\title{
Treatment outcome reporting in anorexia nervosa: time for a paradigm shift?
}

Stuart B. Murray ${ }^{1 *}$, Katharine L. Loeb² and Daniel Le Grange ${ }^{1,3}$

Keywords: Anorexia nervosa, Treatment outcomes, Eating disorders, Precision treatment

Anorexia nervosa (AN) is among the most pernicious of psychiatric disorders, demonstrating a mortality rate six times greater than the general population, and a crude mortality rate of $5-6 \%$ [1]. Even in non-lethal presentations, AN frequently runs a chronic and relapsing illness course, which imparts multi-systemic organ damage, including cardiac abnormalities, structural and functional brain impairment, and bone disease [2]. Alongside these grave medical sequelae, treatment outcomes in AN are universally modest. End-of-treatment remission rates in adolescent AN, the most common period of illness onset, are reported to range from 23 to 33\% [3, 4], of which approximately one third remain in remission at four-year follow-up [5], whereas adult presentations are characterized by end-of-treatment remission rates ranging from 0 to $25 \%$ [6]. The urgent need for novel interventions for $\mathrm{AN}$, as well as augmentations to the potency of existing treatment models, cannot be disputed.

As treatment development studies aim to illustrate and engage specific mechanisms of AN psychopathology, a crucial endeavor lies in precisely indexing the mechanisms of existing treatments, as they relate to the array of symptoms encompassed by AN, including physiological, cognitive and behavioral symptoms. However, existing methods for reporting treatment and course-of-illness outcomes in AN research may have precluded a thorough investigation of both treatment efficacy and mechanisms. Here we outline several important challenges relating to conceptualizations of outcome in AN, which must be considered as we advance towards the development of novel interventions with precise, targeted mechanisms.

\footnotetext{
*Correspondence: stuart.murray@ucsf.edu

'Department of Psychiatry, University of California, San Francisco, 401

Parnassus Avenue, San Francisco, CA 94118, USA

Full list of author information is available at the end of the article
}

\section{The weight- versus cognitive-symptom outcome conundrum}

The symptom profile in AN comprises both physiological and cognitive features, although the central distinction between weight-based versus cognitive symptomatology has historically been underreported in AN treatment trials and long-term course-of-illness studies, with weight status alone being the most widely favoured index of recovery. In studies adopting weight as an exclusive or primary metric of outcome, potentially important differences may go undetected, as may their implications for our understanding of both disorder- and treatment-specific mechanisms. Without question, an essential first step in the treatment of $\mathrm{AN}$ is the reversal of the acute effects of starvation, which may be most readily indexed by weight status. However, inferring from positive increments in our patients' weight that we have effectively engaged the target mechanism of treatment and achieved change in corresponding broader symptom domains, belies the complex and interwoven network of maintaining factors that underpin AN. Moreover, the implicit assumption that weight-based recovery is a proxy for broader cognitive recovery is not supported by evidence, as the constellation of cognitive and affective challenges facing those with AN, including the fear of weight gain, body dissatisfaction, emotional dysregulation, and an ongoing fear of caloriedense foods, frequently persist after significant weight gain has been achieved [7]. Thus, relying on weight outcomes alone in drawing conclusions from randomized controlled trials (RCT) or course-of-illness studies could inadvertently inflate the interpretation of positive results. For instance, long-term naturalistic follow-up data over approximately 20 years illustrate remission rates ranging from $62 \%$ when considering weight status as the sole criterion for a 'good outcome [8], to just $40 \%$ when including both weight and cognitive symptoms [9]. 
More recently, an increasing number of clinical trials have begun to report treatment outcomes as an aggregated function of both weight status and cognitive AN psychopathology, yielding categorical outcome groupings. For instance, "full" remission is typically achieved by attaining both (i) $95 \%$ of expected body weight plus (ii) a score within 1 standard deviation of community norms on gold standard measures of cognitive and behavioural ED psychopathology; "partial" remission can be defined by meeting either of those criteria, but not both; and no remission would reflect an absence of the two criteria. While this approach represents an improvement over exclusively weight-based outcomes, there are potential discrepancies between these component dimensions. Conflating weight-based and cognitive symptom status into unitary outcome measures represents a missed opportunity to elucidate their distinct pathways, which in turn can stymie ongoing attempts to locate precise mechanisms of treatment. Moreover, varying definitions of what constitutes a 'good outcome', even when applied to a single clinical trial, yields remission rates ranging from $2 \%$ - 96\% [10], which has precluded meaningful between-trial comparisons.

\section{Moving forward: Key recommendations}

Accurate indices of potentially differential treatment dimensions, rather than conflated unitary outcomes, are necessary to more closely understand treatment mechanisms. We contest that such delineated outcomes ought to be deemed equally primary to the results and discussion of controlled treatment trials as conflated categorical outcomes, which offer a broad snapshot of outcome. For instance, an RCT may find a significant superiority of one treatment over another in terms of categorical outcomes, yet fail to demonstrate any significant differences across the same treatments in delineated indices of weight or cognitive symptomatology [4]. Interpreting such patterns of results is an important initial step in the development of more potent precision interventions, and is predicated on RCTs systematically reporting results with multiple, and consistent, definitions of outcome. To date in the literature, only a subset of AN studies have done so.

Fully delineating indices of weight- versus cognitive AN symptomatology may not only foster a more nuanced understanding of their respective and combined patterns of change, but also the temporal relationship between them. For instance, examining the latency between nutritional rehabilitation and cognitive relief as a function of moderators, such as duration of illness, will direct our understanding of the intersection between chronicity, the biological effects of starvation, and the mechanistic underpinnings of cognitive AN symptomatology. Alongside these delineated outcomes, incorporating behavioural indicators and parent-report observations of cognitive symptoms will be critical to avoid false negatives in this type of research involving child and adolescent $\mathrm{AN}$ populations with ego syntonic presentations [11].

Finally, rigorous, detailed reporting of longer-term outcomes, as well as treatment engagement during follow up, will allow for more controlled tracking of symptom trajectories and pathways over time. Approximately half of RCTs for AN have reported long-term follow-up data, and of those that do, few have reported whether participants were engaged in ongoing treatment further to completion of the clinical trial, and whether ongoing treatment was concordant or discrepant from the intervention received during the RCT. Ongoing treatment-seeking will by definition be correlated with end-of-treatment symptom status; this means it does not occur at random, and thus represents a confound that compromises the validity of the field's sparse longer-term data on AN.

Clearly, the development of novel treatments for $\mathrm{AN}$ is indicated, although a shift in our conceptualization of treatment outcomes may help identify precise targets for novel interventions. Notably, this may extend beyond weight status and diagnostic cognitive symptoms, i.e., shape and weight disturbance, to include other more transdiagnostic mechanisms such as intolerance of uncertainty and even purported endophenotypic features such as cognitive rigidity [12, 13]. To this end, establishing a benchmark that all treatment studies report independent effect sizes for both weight- and cognitive-based AN symptomatology, alongside indices of the proposed target mechanism of treatment, and details of ongoing treatment engagement among those included in follow-up data, will be a crucial step as the field transitions toward the development of precision treatments. In addition, the field's adherence to an agreed-upon outcome reporting framework that incorporates these recommendations will also facilitate between-trial comparisons, and in turn, more informed practice guidelines for AN.

\section{Funding \\ Dr. Murray receives support from the National Institute of Mental Health (K23MH115184)}

\section{Authors' contributions \\ All authors contributed to the writing and editing of this manuscript. All authors read and approved the final manuscript.}

\section{Authors' information}

Dr. Murray is Assistant Professor in the Department of Psychiatry at the University of California, San Francisco. Dr. Loeb is Professor at Fairlegh Dickinson University. Dr. Le Grange is Endowed Professor at the University of California, San Francisco, and Professor Emeritus at the University of Chicago.

Ethics approval and consent to participate Not applicable.

\section{Competing interests}

Dr. Murray receives royalties from Routledge, and Oxford University Press. Dr. Loeb receives royalties from Routledge, and is a faculty member of and consultant for the Training Institute for Child and Adolescent Eating

Disorders. Dr. Le Grange receives royalties from Guilford Press and Routledge, 
and is co-director of the Training Institute for Child and Adolescent Eating Disorders, LLC.

\section{Publisher's Note}

Springer Nature remains neutral with regard to jurisdictional claims in published maps and institutional affiliations.

\section{Author details}

'Department of Psychiatry, University of California, San Francisco, 401 Parnassus Avenue, San Francisco, CA 94118, USA. ${ }^{2}$ School of Psychology, Fairleigh Dickinson University, Teaneck, NJ, USA. ${ }^{3}$ Department of Psychiatry and Behavioral Neuroscience, The University of Chicago, Chicago, IL, USA.

Received: 31 January 2018 Accepted: 19 April 2018

Published online: 07 May 2018

\section{References}

1. Arcelus J, Mitchell AJ, Wales J, Nielsen S. Mortality rates in patients with anorexia nervosa and other eating disorders: a meta-analysis of 36 studies. Arch Gen Psychiatry. 2011;68:724-31.

2. Mitchell JE, Crow S. Medical complications of anorexia nervosa and bulimia nervosa. Current Opinion in Psychiatry. 2006;19:438-43.

3. Lock J, Le Grange D, Agras WS, et al. Randomized clinical trial comparing family-based treatment with adolescent-focused individual therapy for adolescents with anorexia nervosa. Arch Gen Psychiatry. 2010;67:1025-32.

4. Le Grange D, Hughes EK, Court A, et al. Randomized clinical trial of parent-focused treatment and family-based treatment for adolescent anorexia nervosa. J. Am. Acad. Child Adolesc. Psychiatry. 2016;55:683-92.

5. Le Grange D, Lock J, Accurso EC, et al. Relapse from remission at two- to four-year follow-up in two treatments for adolescent anorexia nervosa. J. Am. Acad. Child Adolesc. Psychiatry. 2014;53:1162-7.

6. Watson HJ, Bulik CM. Update on the treatment of anorexia nervosa: review of clinical trials, practice guidelines and emerging interventions. Psychol Med. 2013:43:2477-500.

7. Fennig S, Klomek AB, Shahar B, et al. A. Inpatient treatment has no impact on the core thoughts and perceptions in adolescents with anorexia nervosa. Early Interv. Psychiatry. 2017:11:200-7.

8. Eddy KT, Tabri TN, Thomas JJ, et al. Recovery from anorexia nervosa and bulimia nervosa at 22-year follow-up. J Clin Psychiatry. 2016;78:184-9.

9. Fichter MM, Quadflieg N, Crosby RD, Koch S. Long-term outcome of anorexia nervosa: results from a large clinical longitudinal study. Int J Eat Disord. 2017;50:1018-30

10. Couturier J, Lock J. What is remission in adolescent anorexia nervosa? A review of various conceptualizations and quantitative analysis. Int J Eat Disord. 2006;39:175-83.

11. Bravender T, Bryant-Waugh R, Herzog D, et al. Classification of eating disturbance in children and adolescents: proposed changes for the DSM-V Eur Eat Disord Rev. 2010;18:79-89.

12. Kesby A, Maguire S, Brownlow R, Grisham JR. Intolerance of uncertainty in eating disorders: an update on the field. Clin Psychol Rev. 2017:56:94-105.

13. Tchanturia K, Anderluh MB, Morris RG, et al. Cognitive flexibility in anorexia nervosa and bulimia nervosa. J Int Neuropsychol Soc. 2004;10(4):513-20.

\section{Ready to submit your research? Choose BMC and benefit from:}

- fast, convenient online submission

- thorough peer review by experienced researchers in your field

- rapid publication on acceptance

- support for research data, including large and complex data types

- gold Open Access which fosters wider collaboration and increased citations

- maximum visibility for your research: over $100 \mathrm{M}$ website views per year

At BMC, research is always in progress.

Learn more biomedcentral.com/submissions 